\title{
Hydroxypropylcellulose coating to improve the biocompatibility and graft-to-bone healing for anterior cruciate ligament reconstruction
}

\author{
Jianjun Yang ${ }^{a}$, Yu Dong ${ }^{b \sharp}$, Jianguang Wang a ${ }^{\sharp}$, Chen Chen ${ }^{\sharp}$, Yuchang Zhu a , Yang Wu ${ }^{b}$, Peng \\ Zhang ${ }^{b}$, Tianwu Chen ${ }^{b}$, Weifeng Zhou d, Peiyi Wu e, Nguyen T. K. Thanh ${ }^{\mathrm{f}}$, Ngọc Quyển Trần $\mathrm{g}$, \\ Jun Chen ${ }^{b *}$ and Shiyi Chen ${ }^{b *}$ \\ 1a Department of Orthopaedics, Tenth People's Hospital of Tongji University, Shanghai 200072, People's \\ Republic of China

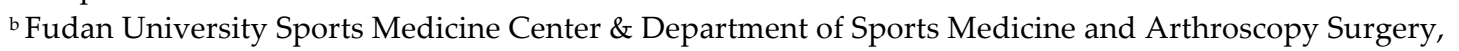 \\ Huashan Hospital, Fudan University, Shanghai 200040, People's Republic of China \\ 'Sports Medicine Center, Shanghai Six People's Hospital \& Six People's Hospital affiliated to Shanghai \\ Jiaotong University, Shanghai 200233, People's Republic of China \\ d Department of Orthopaedics, Cixi People's Hospital \& Cixi Hospital affiliated to Wenzhou Medical \\ University, Ningbo 315300, People's Republic of China \\ e Key Laboratory of Molecular Engineering of Polymers of Ministry of Education, Department of \\ Macromolecular Science and Laboratory of Advanced Materials, Fudan University, Shanghai 200433, \\ People's Republic of China \\ ${ }_{\mathrm{f}}^{\mathrm{f}}$ Biophysics Group, Department of Physics \& Astronomy, University College London, Gower Street, London \\ WC1E 6BT, UK \\ g Department of Materials and Pharmaceutical Chemistry, Institute of Applied Materials Science-VAST, HCM \\ city, Vietnam \\ * Jianjun Yang, Yu Dong, Jianguang Wang and Chen Chen contributed equally to this work. \\ * Correspondence: Dr J. Chen, email: biochenjun@fudan.edu.cn; Prof Shiyi Chen, e-mail: cshiyi@163.com; \\ Tel.: +86-21-52888256
}

\begin{abstract}
Anterior cruciate ligament (ACL) injury is one of the most common injuries in sports, and ACL reconstruction with an artificial ligament is a good treatment for quick recovery. However, current artificial ligaments made of polyethylene terephthalate (PET) are still associated with some problems due to the hydrophobic nature and low biological induction activity of PET. Many efforts have been used to improve the biocompatibility of PET in recent years, and our previous work has shown that surface modification is an effective strategy. Here, a hydroxypropylcellulose (HPC) coating was applied on the surface of a PET artificial ligament order to improve its biocompatibility. The effects of the HPC coating on PET artificial ligament graft-bone healing was investigated in vitro using bone marrow stromal cells (BMSCs), fibroblasts and RSC-364 cells as well as in vivo in a beagle dog model of ACL reconstruction. HPC was coated successfully on the PET and significantly promoted cell growth, adhesion and capability of osteogenic differentiation compared to the PET graft without HPC coating. In vivo, the HPC coating significantly enhanced ligament tissue regeneration. Moreover, the expression of some bone formation- and ligament tissue regeneration-contributing proteins and cell factors, such as COL1, BMP-7, COL3, OCN, RUNX2, TGF- $\beta 1$ and VEGF, was higher on the HPC-coated PET artificial ligament than on the pure PET artificial ligament. In conclusion, HPC coating can significantly improve the cytocompatibility and graft-to-bone healing of a PET artificial ligament for ACL reconstruction.
\end{abstract}

Keywords: tissue engineering; anterior cruciate ligament; polyethylene terephthalate ligament; graft-to-bone healing; surface modification; hydroxypropylcellulose 


\section{Introduction}

The anterior cruciate ligament (ACL), one of four major ligaments of the knee joint, is the most commonly injured ligament in the body. Once it is ruptured by trauma, the injury has very limited ability to heal itself, and surgery is required. ACL reconstruction, in which a graft is used to replace the ligament, is a main surgical procedure for ACL injury; between 60,000 and 175,000 ACL reconstructions are performed yearly in the United States ${ }^{1-2}$. Currently, the grafts commonly used in $\mathrm{ACL}$ reconstruction are autograft and allograft tendons ${ }^{3}$. Whereas the autograft tendon harvest is associated with weakness, pain, and biomechanical alterations at the harvest site, the allograft tendon is associated with infection, disease transmission, and allergic reactions ${ }^{4}$. Therefore, there is a need for the development and application of artificial ligaments that demonstrate favorable safety and biomechanical profiles in ACL reconstruction.

Currently, the Ligament Advanced Reinforcement System (LARS) ligament (Surgical Implants and Devices, Arc-sur-Tille, France), which is made from polyethylene terephthalate (PET), is one of the choices for grafting in ACL injury ${ }^{5}$. Several clinical studies have shown that the LARS ligament possesses several potential postoperative benefits, including the early return to unrestricted sports and strenuous activities, and avoidance of complications caused by using the autografts or allografts, which indicates that it is a good choice for use in ACL reconstruction ${ }^{6-9}$. However, some failure cases have been reported when this artificial ligament is used for reconstructing ACL ${ }^{10-13}$. Recently, we reported a rare case of a 26-year-old patient who suffered from serious synovitis and received LARS artificial ligament reconstruction ${ }^{10}$. We observed a thick fibrous scar tissue around the graft and poorly organized fibrous scar tissue between the graft fibers 3 years later and postulated that these changes might be the causes for the loss of the ligament's structural integrity and the graft's failure ${ }^{10}$. This observation indicates that the currently used LARS artificial ligament leads to poor "ligamentization" in the knee joint and raises questions on how to enhance the healing of the artificial ligament graft after ACL reconstruction.

It is well known that PET is a hydrophobic material, and the hydrophobicity of PET has been reported to account for the observed cases of graft failure ${ }^{10-13}$. In order to overcome the chemical and biological inertness of PET and enhance the graft biocompatibility and bioactivity, some surface modifications are needed ${ }^{14-15}$. In fact, our previous studies have demonstrated that the hydrophilic surfaces of artificial ligament can induce osteoblastic cells to grow well16-20.

In the present study, hydroxypropylcellulose (HPC) was selected due to its bioactive, mucoadhesive and hydrophilic properties ${ }^{19-22}$ and applied to the surface of PET ligaments (shown in Scheme 1). Therefore, the aim of the present study was to fully evaluate the biocompatibility of HPC-coated PET sheets in vitro and in vivo. The regeneration and graft-bone healing of at the HPC-coated PET artificial ligament were assessed by bone marrow stromal cells (BMSCs), fibroblasts and RSC-364 cells in vitro and the tissue in a beagle dog ACL reconstruction model.

A)

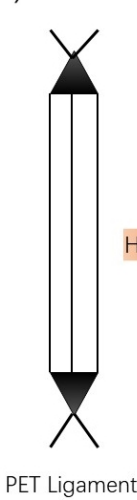

B)

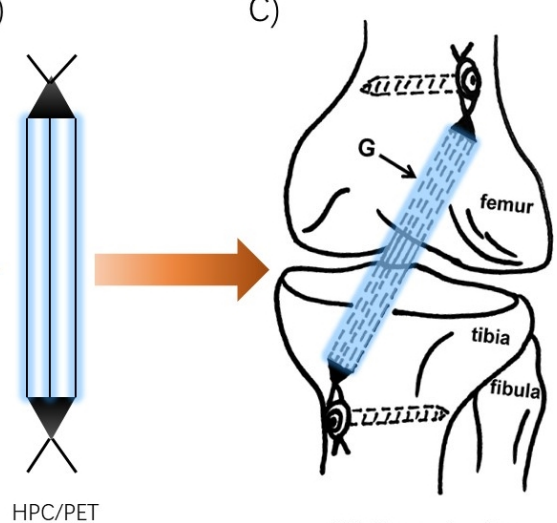

ACL Reconstruction

Scheme.1 The morphology scheme of A) PET ligament, B) HPC/PET ligament. C) The surgical design scheme for the beagle dog ACL reconstruction model using HPC/PET ligament. G, graft. 


\section{Materials and methods}

2.1 Preparation of the PET sheets and artificial ligaments and HPC coating

PET sheets (Fig. 1A) were prepared from a LARS ligament (Surgical Implants and Devices, Arc-sur-Tille, France) and used for experiments in vitro. PET artificial ligaments (Fig. 1B) were made by Donghua University, Shanghai, China for reconstruction of ACL in a beagle dog model. The PET sheets and artificial ligaments were cleansed in $75 \%$ alcohol solution for $4 \mathrm{~h}$, washed with deionized water and then dried in air for $24 \mathrm{~h}$. HPC powder (Klucel ${ }$ LF, Hercules Inc., Wilmington, DE, USA) was dissolved for $1 \mathrm{~h}$ in distilled water by vortexing at room temperature and adjusting the final concentration to $5 \% \mathrm{mg} / \mathrm{L}$, and then left overnight. Then, both PET sheets and artificial ligaments were coated by $\mathrm{HPC}$ in a modified method as previously described ${ }^{19}$. Briefly, the PET sheets and artificial ligaments were undergone the process of corona discharge treatment for $10 \mathrm{~min}$ at first, and then the HPC solution was coated on the surface of PET by using high-pressure spray gun.

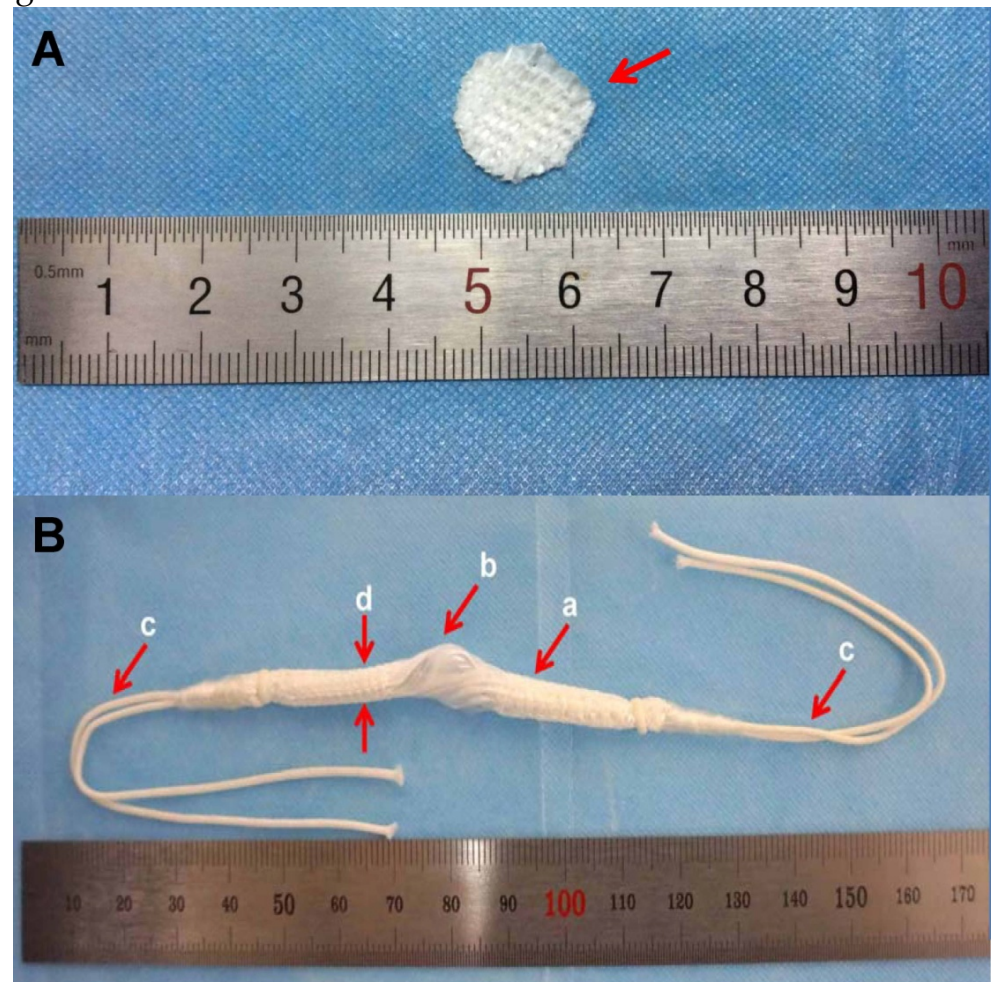

Fig.1. A representative polyethylene terephthalate (PET) sheet from a LARS ligament for in vitro experiments (A) and a representative PET artificial ligament for reconstructing anterior cruciate ligament in beagle dogs (B) with the lengths of the artificial ligament on both sides of the bone tunnel (a), the intra-articular artificial ligament (b) and both ends of the traction line (c) being 4.0, 1.0 and about $10.0 \mathrm{~cm}$, respectively, and the diameter of the artificial ligament on both sides of the bone tunnel $(\mathrm{d})$ being $3.5 \mathrm{~mm}$.

\subsection{Determination of micromorphology and chemicalstructure of PET sheets with HPC coating}

\subsubsection{Scanning electron microscopy (SEM)}

For micromorphological observation, non-coated (PET) and coated (HPC/PET) PET sheets were washed twice with phosphate buffered saline (PBS), and then immersed in PBS containing 1\% glutaraldehyde solution. After $12 \mathrm{~h}$, the sheets were dehydrated in a series of ethanol solutions, i.e. $20 \%, 40 \%, 60 \%, 80 \%$, and $100 \%$, followed by $24 \mathrm{~h}$ of air-drying. The PET and HPC/PET sheets were gold sputtered under vacuum and subjected to examination by SEM (TS5136MM; Tescan, Brno, Czech Republic).

\subsubsection{Fourier transform infrared spectroscopy (FTIR)}

PET sheets were immersed in 10\% HPC solution for $24 \mathrm{~h}$ and dried in an oven for $24 \mathrm{~h}$. Then, the PET and HPC/PET sheets were examined with FTIR on a Nicolet Nexus 470 spectrometer at a 
spectral resolution of $4 \mathrm{~cm}^{-1}$ using 128 scans. The preconditioning process of FTIR, including baseline correction and spectral peak reading, was performed with OMNIC 8.0 software (Thermo Nicolet Corp., Waltham, USA). The test was performed three times to ensure the repeatability and reliability of the data.

\subsection{Cell experiments in vitro}

\subsubsection{Sources and culture of bone marrow stromal cells (BMSCs), fibroblast cells and RSC-364cells}

Bone marrow stromal cells (BMSCs) were isolated from five 8-week-old male New Zealand white rabbits $(2.5 \pm 0.3 \mathrm{~kg})$, Department of Laboratory Animal Science, Fudan University, Shanghai, China) according to procedures described previously. ${ }^{23}$ The rabbit ACL fibroblasts were prepared by the explant culture method, and RSC-364 cell line (Shine Star Biological Engineering Co., Ltd., Hubei, China) was purchased. These three kinds of cells were cultured in Dulbecco's modified Eagle's medium (DMEM) in a $5 \% \mathrm{CO}_{2}$ incubator at $37{ }^{\circ} \mathrm{C}$ before the experiments, as described below.

\subsubsection{Cell counting kit-8 (CCK-8) assay for proliferation activity of BMSCs, fibroblast and RSC-364 cells}

CCK-8 assay (CCK-8, Dojindo, Japan) was used to determine cell proliferation. Briefly, a total of $1 \times 10^{4}$ cells (BMSCs, fibroblast or RSC-364 cells) per well were seeded into a 96-well plate, inside which ligaments were preset. After 1, 2, 4, 6, 8 and $10 \mathrm{~d}$ of culture, $10 \mu \mathrm{L}$ CCK-8 solution was added to each well, and the plate was incubated at $37^{\circ} \mathrm{C}$ for $4 \mathrm{~h}$. Then, $100 \mu \mathrm{L}$ culture supernatant was collected and added to another 96-well plate, which was read on a microplate reader (Thermo Nicolet Corp., Waltham, USA) for absorbance at $450 \mathrm{~nm}$. The experiment was performed in triplicate, and the average of the results was calculated.

\subsubsection{SEM for detection of cell adhesion on PET sheets or HPC/PET sheets}

PET sheets and HPC/PET sheets $(1.5 \mathrm{~cm}$ diameter) were placed in $70 \%$ ethanol for $1 \mathrm{~h}$ and stayed in a laminar-flow cabinet overnight to dry. The sheets were washed twice with sterile PBS, placed into a 24-well plate, and then incubated in the basic cell culture medium in a humid $5 \% \mathrm{CO}_{2}$ incubator at $37{ }^{\circ} \mathrm{C}$ for $4 \mathrm{~h}$. The three kinds of cells were then cultured in DMEM for $4 \mathrm{~h}$ at $37^{\circ} \mathrm{C}$ in a $5 \% \mathrm{CO}_{2}$ incubator. After removal of the medium, cells $\left(5 \times 10^{4} / \mathrm{ml}\right)$ in the logarithmic phase were cultured in the basic cell culture medium containing stainless PET sheets for up to $7 \mathrm{~d}$.

SEM was used to observe the morphology of BMSCs, fibroblast and RSC-364 cells cultured for $7 \mathrm{~d}$. The PET sheets and cultured cells were washed three times with PBS and fixed in 1\% glutaraldehyde solution for $12 \mathrm{~h}$. After washing with PBS 3 times, they were dehydrated in a series of ethanol solutions, i.e., $20 \%, 40 \%, 60 \%, 80 \%$, and $100 \%$, and dried in a vacuum for $24 \mathrm{~h}$. Samples were finally coated with gold palladium and observed with SEM.

2.3.4 Western blotting for expression of integrin- $\beta 1, B M P-2$ and $B M P-7$ in vitro

BMSCs, fibroblasts and RSC-364 cells were used for the determination of integrin- $\beta 1$ expression, and BMSCs were used for the determination of BMP-2 and BMP-7 expression. In brief, cells $\left(1 \times 10^{5}\right)$ were seeded into 6-well plates, inside which PET sheets were preset, and cultured under conditions as described above for $7 \mathrm{~d}$ and collected. Total proteins were then extracted, and the quantity was determined using the Pierce ${ }^{\mathrm{TM}}$ bicinchoninic acid protein assay kit (Thermo Fisher Scientific, Hudson, USA). After denature at $95^{\circ} \mathrm{C}$ for $10 \mathrm{~min}$, the protein samples were separated by SDS-PAGE on a separation gel of $11 \%$, and then transferred to a PVDF membrane (Millipore, WI, USA) at $400 \mathrm{~mA}$. The blots were blocked in Tris-buffered saline with Tween 20 containing $5 \%$ nonfat milk for $2 \mathrm{~h}$ at room temperature, and incubated overnight with the primary antibodies (dilution: GAPDH, 1: 1000; integrin- $\beta 1,1: 800$; BMP-2 and BMP-7, 1:500) at $4{ }^{\circ} \mathrm{C}$. Then the blots were incubated with the secondary antibodies (1: 4000). The bands were developed by enhanced chemiluminescence substrates (Thermo Nicolet Corp., Waltham, USA) using X-ray sheets, and then scanned and quantified by densitometric analysis using GAPDH as a reference.

2.3.5 Enzyme-linked immunosorbent assay (ELISA) for the levels of alkaline phosphatase (ALP) and osteopontin (OPN) in vitro

The levels of ALP and OPN were carried out using BMSCs in a 96-well plate by ELISA, according to the manufacturer's specifications (Abcam, CA, USA). BMSCs $\left(2 \times 10^{4}\right)$ were seeded in each well of a 24-well plate, inside which ligaments were preset; each well contained DMEM with 
$10 \%$ fetal bovine serum. After $5,7,10$ and $14 \mathrm{~d}$ of culture, cells were collected for determination of expression levels of ALP and OPN.

\subsection{Animal experiments}

\subsubsection{Ethical consideration and establishment of ACL reconstruction model in beagle dogs}

The Institutional Animal Care and Use Committee at Fudan University approved the protocols for all animal experiments involving rabbits and beagle dogs, and all animal experiments were conducted following the NIH Guide for the Care and Use of Laboratory Animals.

Twenty-four 12-month old male healthy beagle dogs from Shanghai Sixth People's Hospital, weighing an average of $12.40 \pm 1.58 \mathrm{~kg}$ were randomly selected to receive ACL reconstruction with artificial ligament grafts with $(n=12)$ or without $(n=12)$ HPC coating. During the study period, they were kept in cages $(120 \mathrm{~cm} \times 100 \mathrm{~cm} \times 100 \mathrm{~cm})$, and their activities were limited only in the cages.

The animal surgical procedures for ACL reconstruction model were undertaken as previously described ${ }^{23}$. Briefly, animals were intravenously injected with pentobarbital sodium $(30 \mathrm{mg} / \mathrm{kg})$. ACL reconstruction was performed on one knee at random. Through an anteromedial incision and a medial parapatellar arthrotomy, the knee was exposed and the native ACL was excised. The tibial tunnel was first created according to the width of the distal part of the graft, which was normally $4.5 \mathrm{~mm}$, with a $45^{\circ}$ angle to the tibial axis and a $15^{\circ}$ to $30^{\circ}$ angle to the sagittal plane. The femoral tunnel, with a width of $4.5 \mathrm{~mm}$ for the whole length, was created through the tibial tunnel in $70^{\circ}$ to $90^{\circ}$ knee flexion by the use of a guide wire and a $4.5-\mathrm{mm}$ cannulated drill. The artificial ligament graft was pulled into the bone tunnel for ACL reconstruction, and the two ends of the graft were fixed by the through-passing and in-braided sutures over post screws on the outer aspects of the distal femur and proximal tibia, respectively (Supplementary Fig. 1). The wound was then closed in layers.

Postoperatively, the dogs were not immobilized, and each dog was allowed free activity in its own cage. The incision site was cleansed with benzalkoniumchloride every $3 \mathrm{~d}$. Intramuscular injection with penicillin $(3,200,000 \mathrm{U})$ and streptomycin $(1 \mathrm{~g})$ was administered every day for the first 3 postoperative days. Six dogs in each group were sacrificed by a lethal injection of pentobarbital 4 and $16 \mathrm{wk}$ after surgery, respectively: three for mechanical testing, and three for histological and immunohistochemical examinations and real-time polymerase chain reaction (RT-PCR).

\subsubsection{Mechanical testing}

After sacrifice, the tibia-graft-femur complex was immediately prepared for mechanical testing. The scar tissues were removed with caution from the tibial and femoral tunnel exits, and all the screws were removed from the distal femur and proximal tibia before mechanical testing. During the testing, the surrounding soft tissues were dissected and the posterior cruciate ligament (PCL) was released. Thus, only the reconstructed ACL remained to connect the tibia and femur. Then, the knee joints were mounted on an Instron materials testing system machine (5569; Instron Co., Norwood, MA, USA) for pullout testing at $20{ }^{\circ} \mathrm{C}$ and a humidity of $65 \%$. The tibia-graft-femur complex was preconditioned with a static preload of $10 \mathrm{~N}$ for $5 \mathrm{~min}$. Then, the maximum failure load, defined the real-time recorded stress that is reduced to $50 \%$ of the stress peak value, and the stiffness of limbs with PET artificial ligaments, referring to the ability of the material to resist elastic deformation when the material is stressed, were immediately determined using an elongation rate of $2 \mathrm{~mm} / \mathrm{min}$. The test for each sample was considered completed when the graft ruptured or was pulled out of the bone tunnel. During the testing, the bone tunnels and graft were adjusted in one line and the traction force was applied along this line.

\subsubsection{Histological examination}

After sacrifice, the tibia-graft-femur samples were immediately cut and fixed in $10 \%$ formalin, and embedded in paraffin wax $48 \mathrm{~h}$ later. Then, the samples were sectioned ( $5 \mu \mathrm{m}$ thick) perpendicular to the graft's longitudinal axis. Sections prepared from the PET artificial ligament at both ends of the bone tunnel were treated with Masson trichrome stain for histological evaluation of collagen levels and osteogenesis in the graft-tibia or -femur complex. An inverted light microscope (IX71SBF-2; Olympus, Tokyo, Japan) was used to visualize the Masson trichrome stain 
images, and a DP Manager (Olympus Optical Co., Tokyo, Japan) was used to capture the digital images.

2.4.4Immunohistochemistry for COL1 expression

The sections of PET artificial ligament in the articular cavity were deparaffinized and rehydrated. Endogenous peroxidase was blocked for $30 \mathrm{~min}$ with $0.3 \%$ hydrogen peroxide at $37^{\circ} \mathrm{C}$, and further for $20 \mathrm{~min}$ with a blocking reagent (PBS containing goat serum). The sections were washed twice with PBS buffer and incubated with the primary antibody (rabbit anti-dog COL1, dilution 1: 50, Bioworld technology co Ltd., Nangjing, China) at $4{ }^{\circ} \mathrm{C}$ overnight. After washing, the sections were incubated with a secondary antibody (MaxVision kit, Maixin Biotechnology, Fuzhou, China) for $15 \mathrm{~min}$ at room temperature, and then treated with dimethylaminoazobenzene (Simple Stain DAB Solution, Maixin Biotechnology, Fuzhou, China) for $5 \mathrm{~min}$, followed by counter-staining with hematoxylin. A DP Manager (Olympus Optical Co., Tokyo, Japan) was used to obtain the digital images, and Image-Pro Plus 6.0 software (Media Cybernetics Corp., Rockville, USA) was used to measure COL1 expression, which was expressed as the mean area value of COL1-positive staining at the graft site with $200 \times$ magnification.

\subsubsection{RT-PCR}

After sacrifice, the graft-tibia or -femur complex at each end of the bone tunnel was harvested from each knee and subjected to RT-PCR. Briefly, total RNA from the interfacial tissues between the host bone tunnel and graft were prepared using TRIzol reagent (10296010; Invitrogen, Carlsbad, CA, USA), and cDNA was prepared using reverse transcriptase MMLV (D2640A; Takara, Beijing, China). SYBR Premix Ex Taq (DRR041A; Takara, Beijing, China) was used for quantitative PCR, and an RT-PCR system (TP800; Takara, Kyoto, Japan) was used to detect mRNA expression of BMP-7, COL3, OCN, RUNX2, TGF- $\beta 1$ and VEGF, which was normalized to that of $\beta$-actin. All the primers used in RT-PCR are listed in Table 1.

Table 1 The primers for RT-PCR

\begin{tabular}{|c|c|c|}
\hline & Forward & Reverse \\
\hline$\beta$-actin & $\begin{array}{l}\text { 5'-CCA AGG CCA ACC GCG } \\
\text { AGA AGA TGA-3' }\end{array}$ & $\begin{array}{l}\text { 5'-GCA GCG CGTAGC CCT CGT AGA } \\
\text { TGG-3' }\end{array}$ \\
\hline$B M P-7$ & $\begin{array}{l}\text { 5'-GAG AGA GAC CTG CTG } \\
\text { GGT TG-3' }\end{array}$ & 5'-AGA ACT GCC TTG GTT GCA GT-3' \\
\hline COL3 & $\begin{array}{l}\text { 5'-TGA GGG CCA AAG CGA } \\
\text { AGA CAT CC-3' }\end{array}$ & $\begin{array}{l}\text { 5'-TTC GGG GCA GAC AGG GCA GCA } \\
\text { CTC-3' }\end{array}$ \\
\hline OCN & $\begin{array}{l}\text { 5'-GATGCTGCAGTTGAATGTC } \\
\text { TG-3' }\end{array}$ & 5'-CAG AAC AGC TCT CCA CAG CA-3' \\
\hline RUNX2 & $\begin{array}{l}5^{\prime} \text {-TCT TAC CCC TCC TAC } \\
\text { CTG AGC-3' }\end{array}$ & 5'-ACG TCG GTG ATG ACA GGA A-3' \\
\hline TGF- $\beta 1$ & $\begin{array}{l}\text { 5'-GCC AGA TCC TGT CCA } \\
\text { AGC TG-3' }\end{array}$ & 5'-GGT GAC CTC CTT GGC GTA GTA-3' \\
\hline VEGF & $\begin{array}{l}\text { 5'- CGG GAA CCA GAT CTC } \\
\text { TCA CC }-3^{\prime}\end{array}$ & 5'-AAA ATG GCG AAT CCA ATT CC-3' \\
\hline
\end{tabular}

Abbreviations: BMP-7, bone morphogenetic protein-7; TGF- $\beta 1$, transforming growth factor- $\beta 1$; COL3, type III collagen; VEGF, vascular endothelial growth factor; OCN, osteocalcin.

\section{Statistical analysis}

Numerical data were expressed as the mean \pm standard deviation (SD). Anon-paired t-test and was used to compare the data of the experimental and control groups, and an independent two-sample Student's t-test was used to compare groups at various time points. Statistical analyses were performed using the SPSS for Windows v. 13.0.0 statistical software (SPSS, Inc., USA). AP value of $<0.05$ was considered statistically significant.

\section{Results}

3.1 Micromorphology and chemical structure of PET sheets and HPC/PET sheets 
The morphology of PET and HPC/PET sheets were observed by SEM( Fig. 2). The surface of the PET sheets was smooth (Fig. 2A), while a thin HPC layer was appeared on the fiber surface of the HPC/PET sheets (Fig. 2B).

The FTIR results confirmed that HPC was modified on the surface of PET, and there were also some characteristic peaks for HPC coating, including O-H stretching vibration (3600-3200 $\left.\mathrm{cm}^{-1}\right)$ and $\mathrm{C}-\mathrm{H}$ stretching vibration $\left(3011-2819 \mathrm{~cm}^{-1}\right)$ in the HPC/PET sheets in comparison with the pure PET sheets (Fig. 2C).

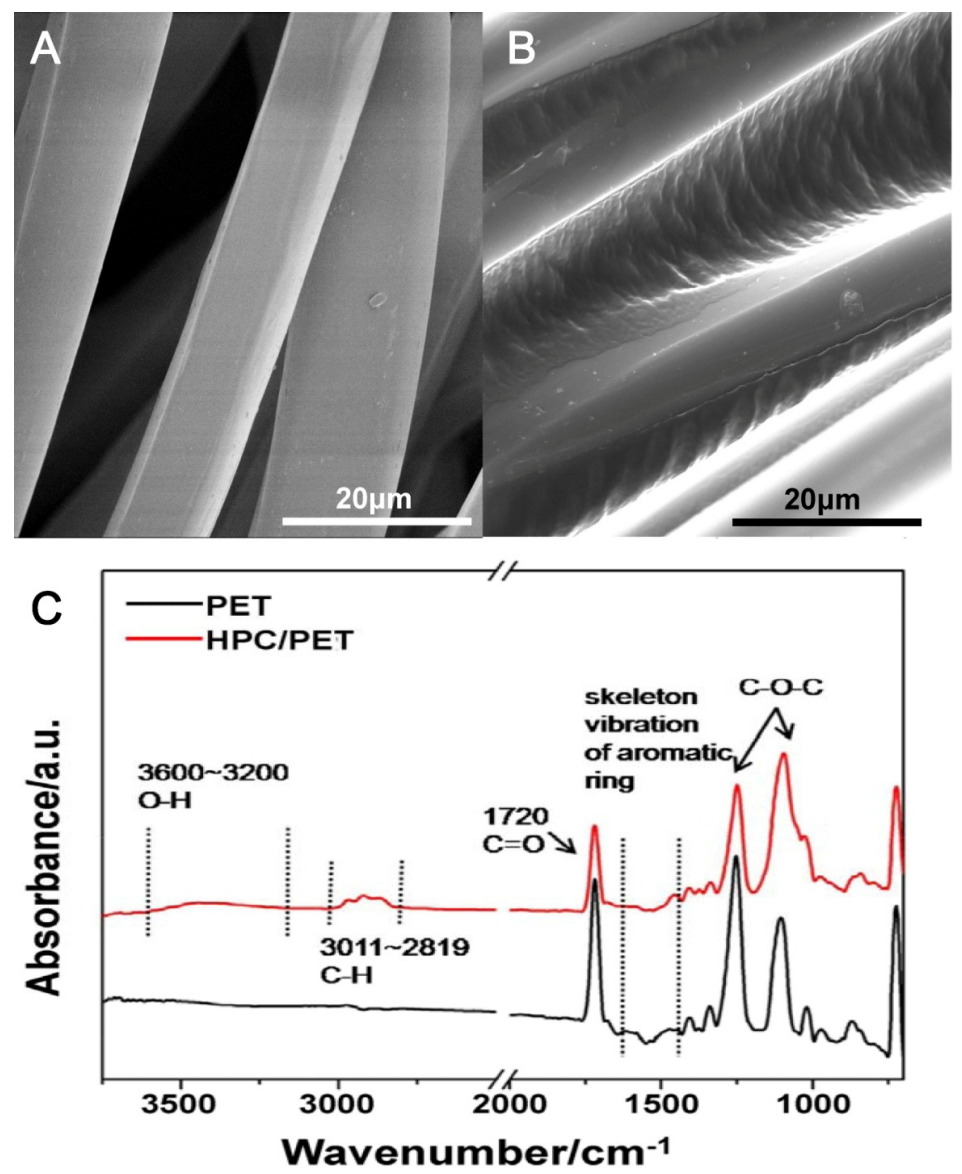

Fig. 2. Micromorphology of the surfaces of the PET sheets (A) and HPC/PET sheets (B), as detected by SEM, and the spectra of the PET sheets and HPC/PET sheets, as detected by FTIR. PET, polyethylene terephthalate; HPC, hydroxypropylcellulose; $\mathrm{Bar}=20 \mu \mathrm{m}$.

\subsection{Cell proliferation of BMSCs, fibroblasts and RSC-364 cells in vitro}

The observed viability of BMSCs, fibroblasts, and RSC-364 cells on the PET and HPC/PET sheets, as assessed by CCK-8 assay, is presented in Fig. 3. The OD value at A450 nm was taken as the reference value for cell viability. The results of CCK-8 assay showed that the cell viability of BMSCs cultured with HPC/PET sheets at 6, 8 and $10 \mathrm{~d}$ was significantly higher than that of those cultured with PET sheets. There was no significant difference in the value of cell viability between BMSCs cultured with HPC/PET sheets and those cultured with PET sheets for 1, 2 and $4 \mathrm{~d}$ (Fig. 3A).

Furthermore, there was no significant difference in the cell viability of fibroblasts between the two groups after 1 and $2 \mathrm{~d}$ of culture (Fig. 3B). However, fibroblasts cultured with HPC/PET sheets for 4, 6, 8 and $10 \mathrm{~d}$ had a significantly higher value for cell viability than those cultured with PET sheets.

Moreover, the cell viability of RSC-364 cells cultured on the surface of PET and HPC/PET sheets also showed no significant difference at 1, 2, 4 and $6 \mathrm{~d}$, while a significant difference in two group was observed at 8 and $10 \mathrm{~d}$ (Fig. 3C). 

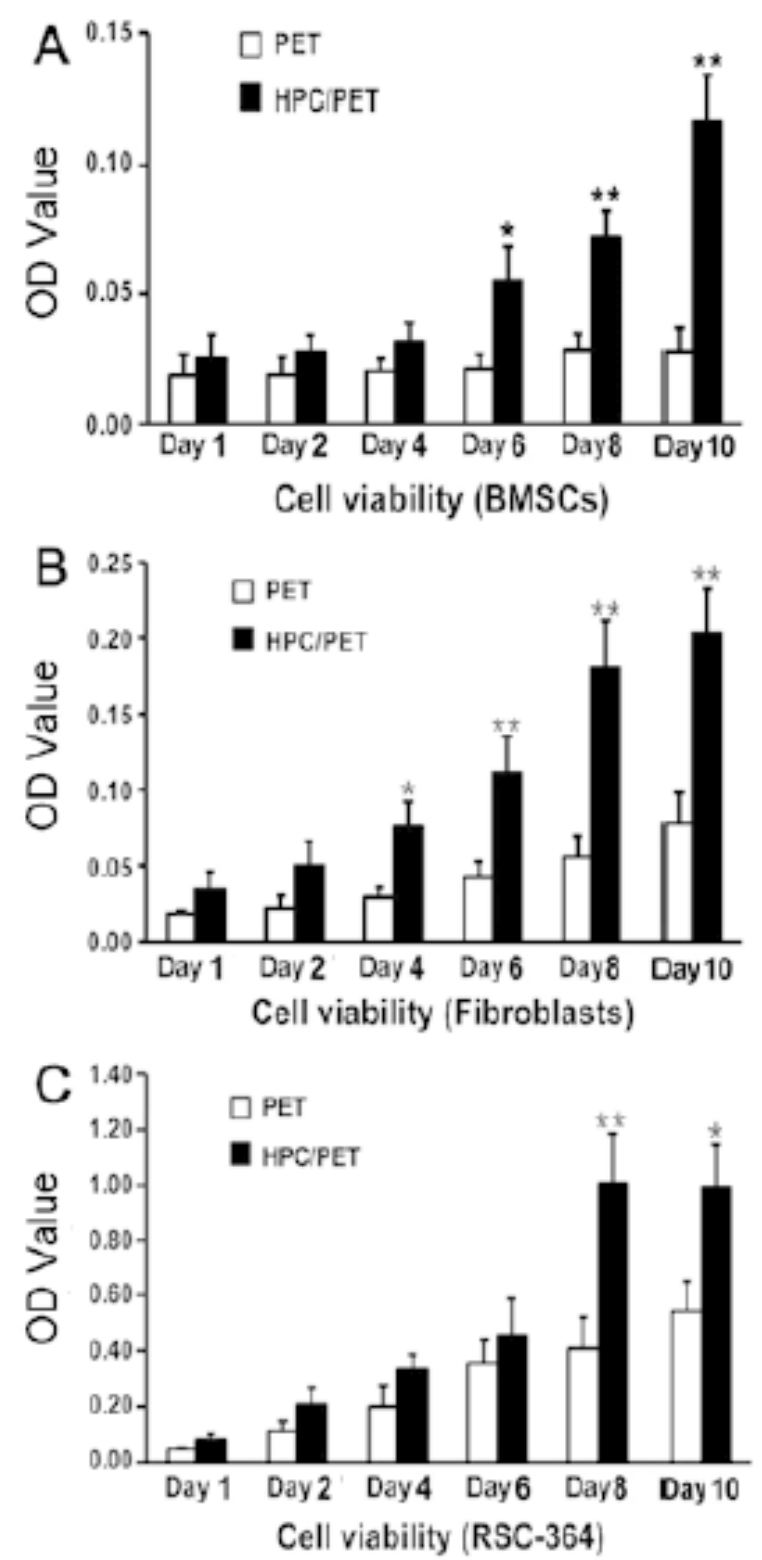

Fig. 3. Proliferation of bone marrow stromal cells (A), fibroblasts (B) and RSC-364 cells (C) as detected by CCK- 8 assay at various time points in culture with HPC/PET sheets and PET sheets. PET, polyethylene terephthalate; HPC, hydroxypropylcellulose. ${ }^{*}, P<0.05 ;{ }^{* *}, P<0.01$.

\subsection{Adhesion and expression of integrin- $\beta 1$ of BMSCs, fibroblasts and RSC-364 cells}

The SEM images about the cell morphology on the surface of both PET and HPC/PET sheets were shown in Fig. 4. The BMSCs, fibroblasts and RSC-364 cells rarely adhered onto the PET sheets after $7 \mathrm{~d}$ of culture, respectively (Fig. 4A, D, and G). However, all of tested cell including BMSCs, fibroblasts and RSC-364 cells extensively grown onto the HPC/PET sheets, respectively (Fig. 4B and $\mathrm{C}, \mathrm{E}$ and $\mathrm{F}$ and $\mathrm{H}$ and $\mathrm{I}$ ), and exhibited polygon shapes with multiple cellular synaptic contacts (Fig. 4). 

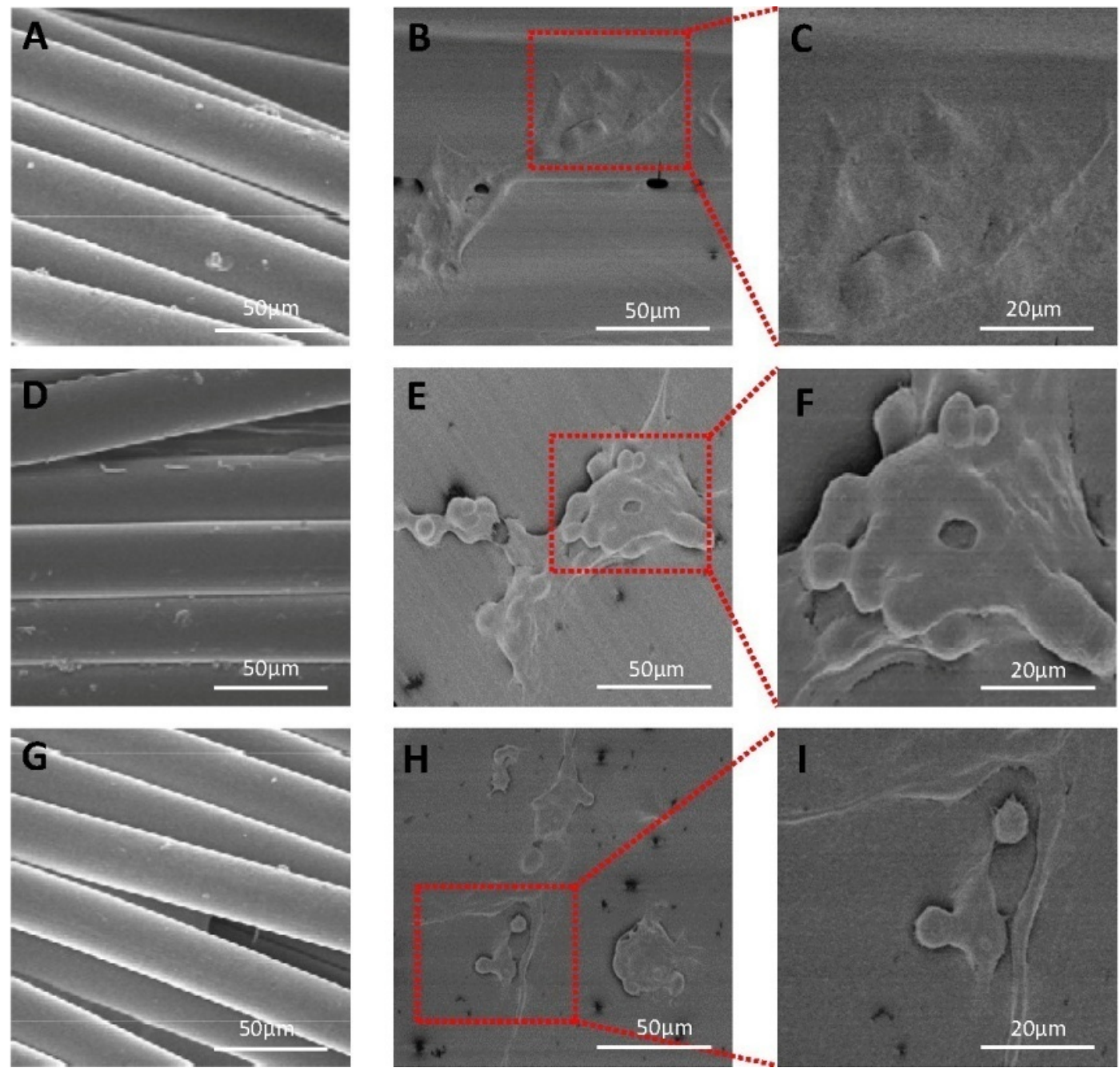

Fig. 4. SEM showing the morphology and adhesion of BMSCs on the PET sheets (A) and HPC/PET sheets (B, C), fibroblasts on the PET sheets (D) and HPC/PET sheets (E, F) and RSC-364 cells on the PET sheets $(\mathrm{G})$ and HPC/PET sheets $(\mathrm{H}, \mathrm{I})$ after $7 \mathrm{~d}$ of culture. SEM, scanning electron microscopy; BMSCs, bone marrow stromal cells.

\subsection{Expression of proteins related to osteogenic differentiation in BMSCs}

The protein expression levels of integrin- $\beta 1$ in BMSCs, fibroblasts and RSC-364 cells were determined by western blotting (Fig. 5). The result of western blotting showed that integrin- $\beta 1$ expression in HPC/PET sheets was higher than that in the corresponding cells cultured with PET sheets $(0.4756 \pm 0.0567$ vs. $0.1812 \pm 0.0173, p=0.001 ; 0.5722 \pm 0.4960$ vs. $0.3346 \pm 0.0418, p=0.003$; and $0.6316 \pm 0.0394$ vs. $0.4175 \pm 0.0368, p=0.002$, respectively) (Fig. 5). 


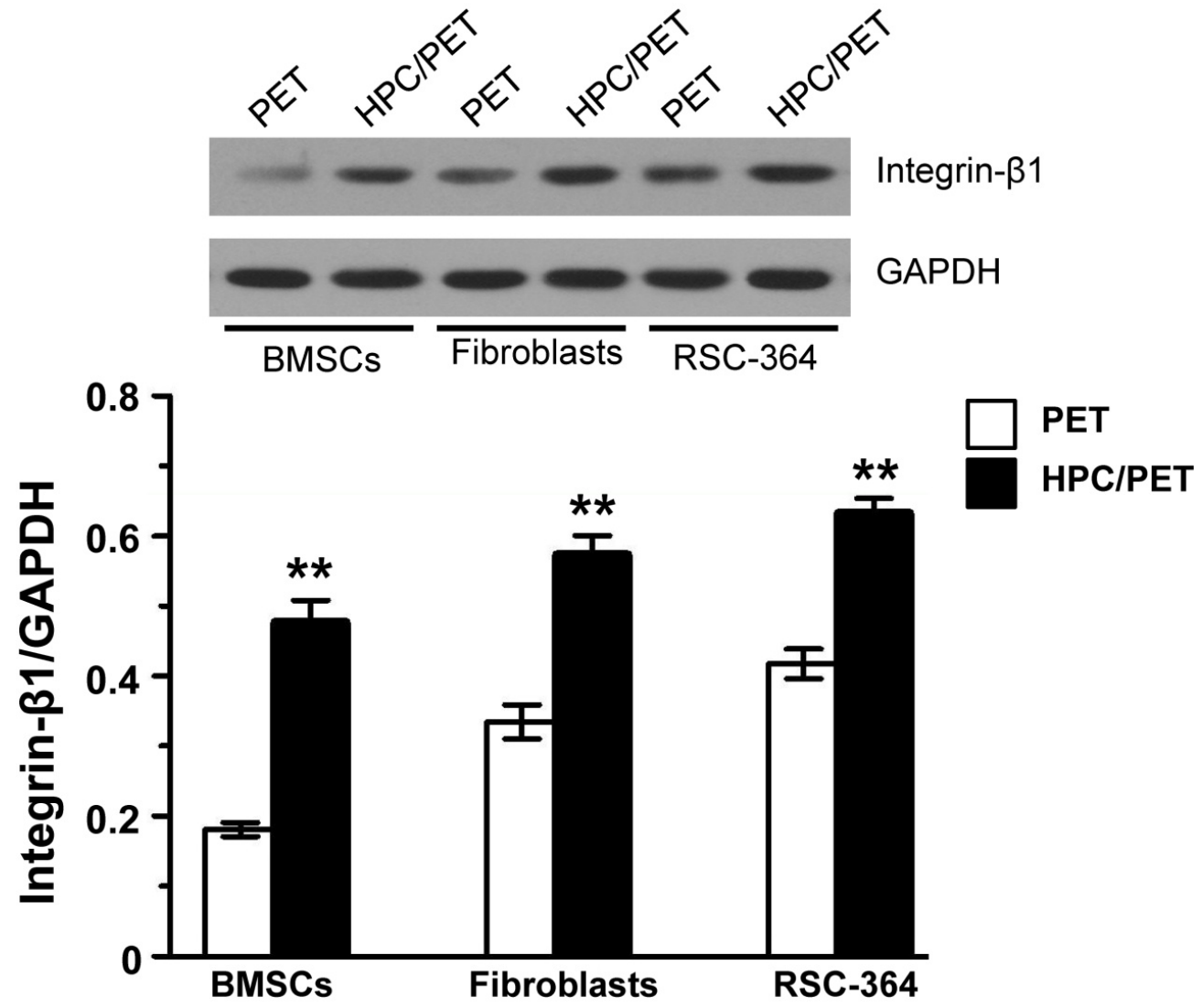

Fig. 5. Protein expression of integrin- $\beta 1$ in BMSCs, fibroblasts and RSC-364 cells cultured with PET sheets and HPC/PET sheets for $7 \mathrm{~d}$. ${ }^{* *} P<0.01$.

Furthermore, BMP-2 and BMP-7 were up-regulated in BMSCs cultured with HPC/PET sheets for $7 \mathrm{~d}$ in comparison with levels in the PET sheets group (Fig. 6A\&6B). Moreover, both the ALP and OPN levels were further assessed by ELISA. As shown in Fig. 6, both the expression levels of OPN and ALP on BMSCs cultured with HPC/PET sheets were up-regulated with increasing time. Notably, both OPN and ALP expression levels in HPC/PET sheets were significantly higher compared with those on pure PET sheets beginning at $5 \mathrm{~d}$ (Fig. 6C and D).
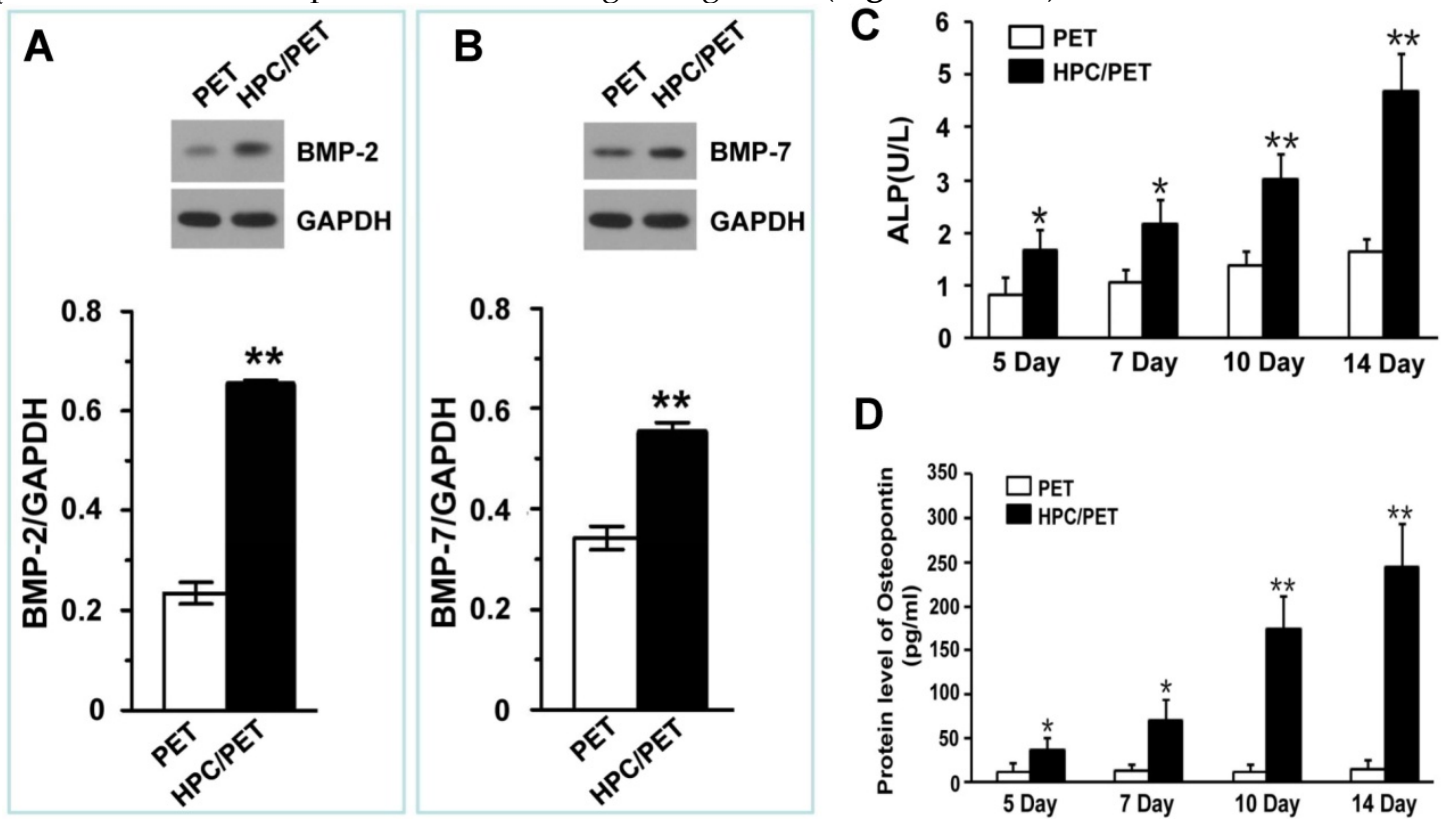

D

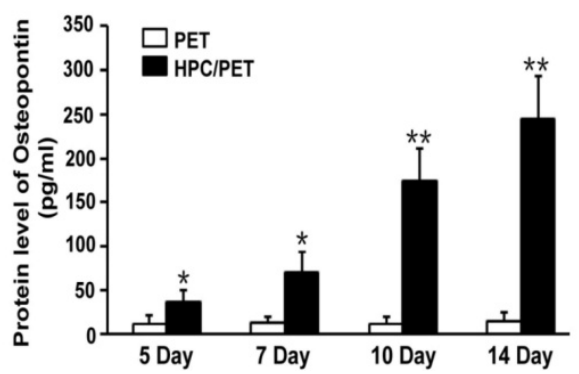

Fig. 6. Protein levels of BMP-2 (A) and BMP-7 (B), as determined by western blotting and ALP (C) and osteopontin (D), as detected by ELISA, in BMSCs cultured with PET sheets or HPC/PET sheets for $7 \mathrm{~d}$ at indicated time points.. ${ }^{*}, P<0.05 ;{ }^{* *}, P<0.01$. 


\subsection{In vivo evaluation of PET artificial ligament grafts in ACL reconstruction beagle model}

Fig. 7 shows the results of PET artificial ligament grafts in ACL reconstruction beagle model. As shown in Fig 7A-D, all knees in the beagle dogs were good without severe postoperative complications after a long period, even $16 \mathrm{wk}$. Furthermore, all the grafts still remained good mechanical capability, as they were not broken and there was no loosening of any fixation screws.

As shown in Fig. 7A and B, there was joint effusion surrounding the graft in the articular cavity, with thick and rough soft tissue on the PET graft at $4 \mathrm{wk}$ after implantation, while a thin synovium covering appeared on the surface of the HPC/PET graft. Meanwhile, although a thin synovium was also observed on the PET graft after $16 \mathrm{wk}$, a much thicker synovium covered the whole part of HPC/PET graft (Fig. 7C and D).

Immunohistochemistry showed that COL1 expression in the articular cavity was higher in the HPC/PET graft than in the PET graft at weeks 4 and 16 (693.67 \pm 45.98 vs. $516.67 \pm 37.61$ at week 4, $P$ $=0.007$; and $732.33 \pm 49.62$ vs. $550.00 \pm 43.49$ at week 16, $P=0.009$ ) (Fig. 7E-7H). In addition, the expression levels of COL1 increased with time in both groups (Fig. 7).

\section{PET}
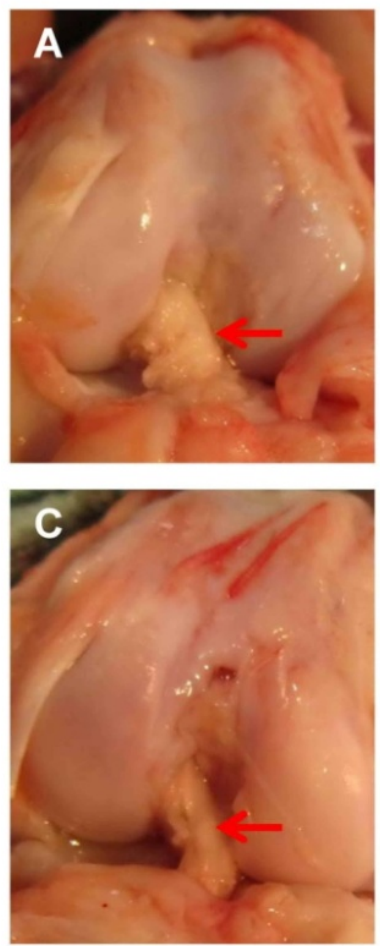

HPC/PET
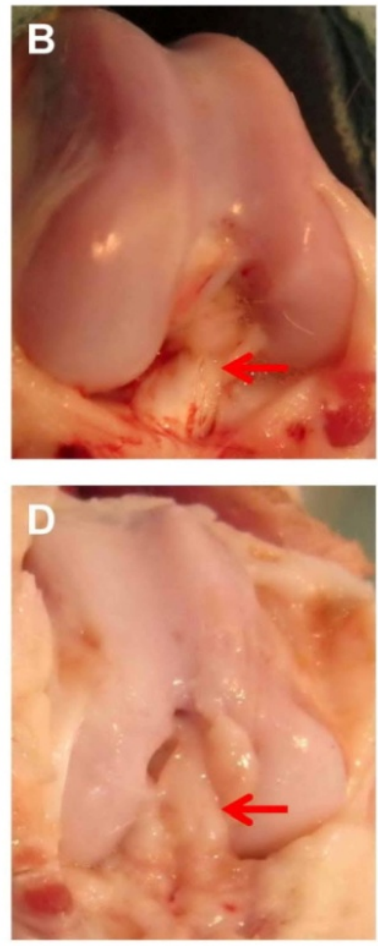

PET
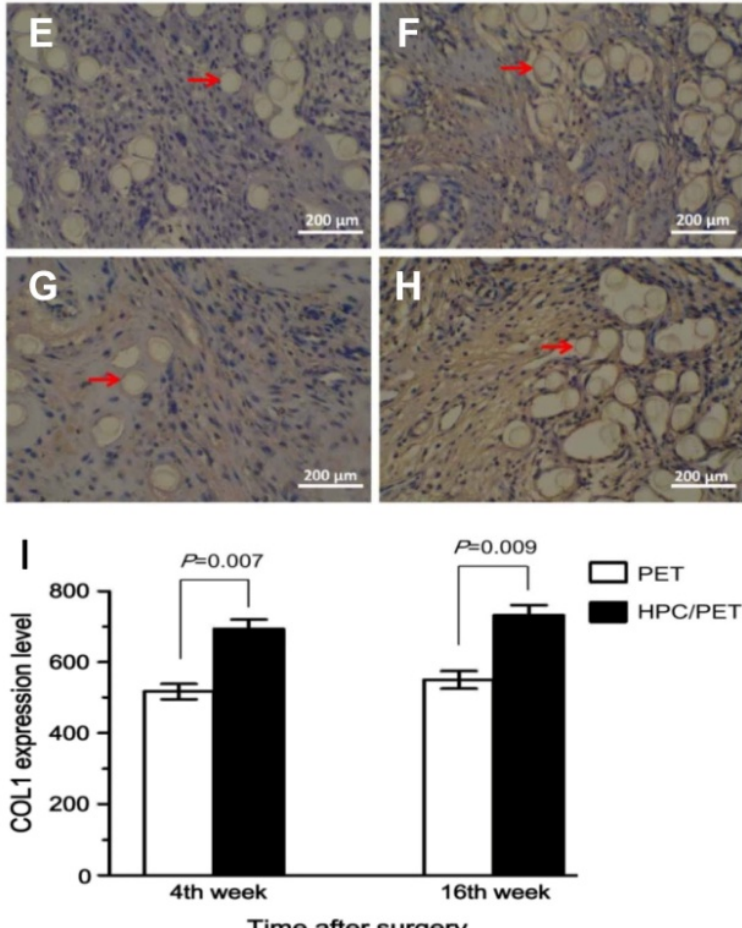

Fig. 7. A-D. Gross observation of the PET artificial ligament grafts and HPC/PET artificial ligament graft in the articular cavity 4 (A and B, respectively) and 16 (C and D, respectively) wk after surgery. E-I. Immunohistochemical analysis showing the expression levels of COL1 in the PET grafts and HPC/PET grafts in the articular cavity $4 \mathrm{wk}$ (E and F, respectively) and $16 \mathrm{wk}$ (G and H, respectively) after surgery, with the corresponding quantitatively measured levels of COL1 (I). Red arrow shows the PET artificial ligament grafts. Bar $=200 \mu \mathrm{m}$. 

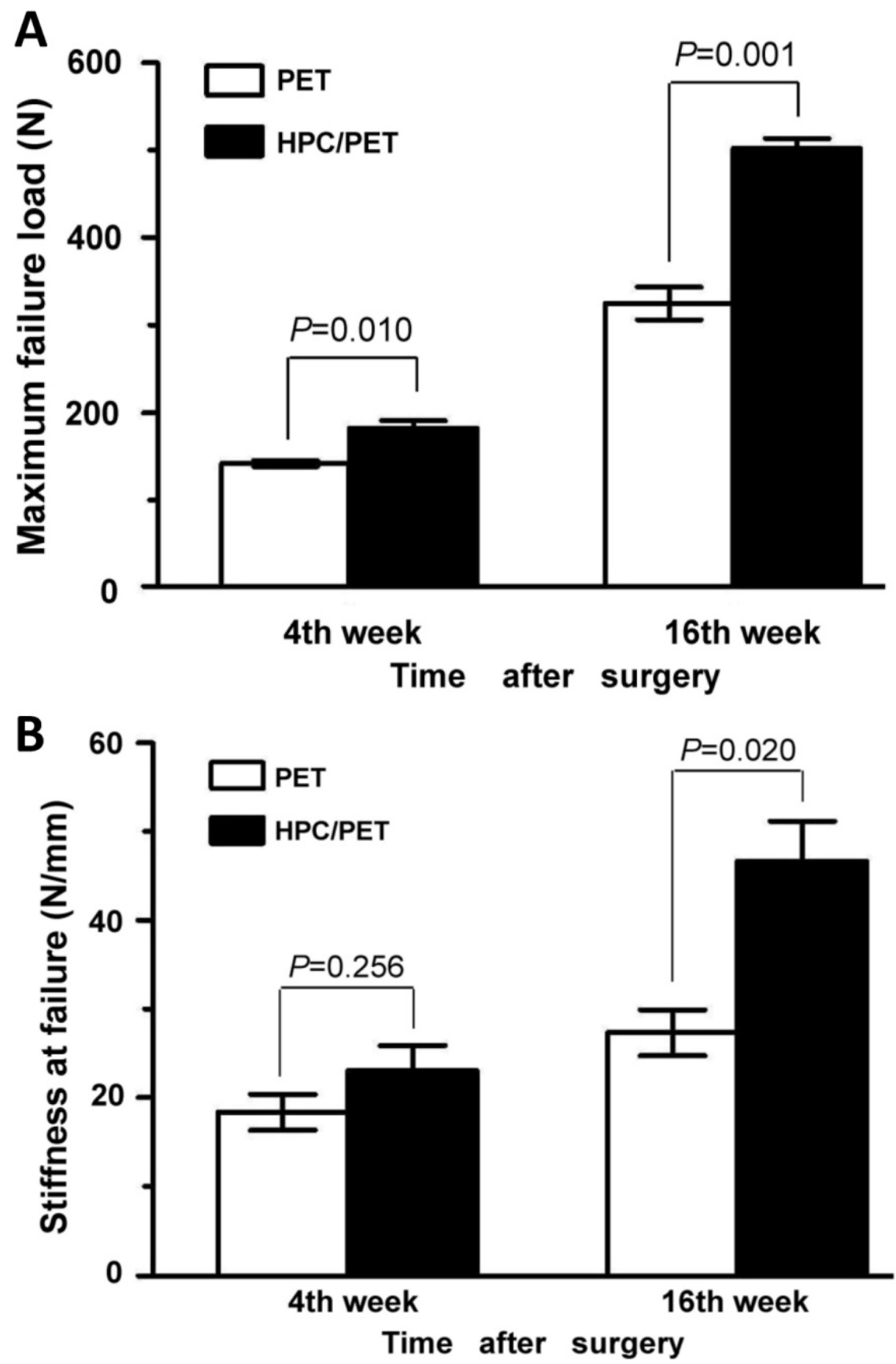

Fig. 8. Mechanical graft-bone healing examinations in beagle dog model at various time points after surgery. (A) Comparison of maximum failure load between the PET group and HPC/PET artificial ligament group. (B) Comparison of stiffness at failure between the PET and HPC/PET artificial ligament groups.

3.6 Maximum failure load and stiffness of the limbs with PET artificial ligaments

The mechanical abilities of implants after 4 and 16 wk were detected (Fig. 8). All PET grafts were pulled out without difficulty from the bone tunnel following removal of the screws 4 and 16 wk after the surgery. No PET artificial ligaments were ruptured. At 4 and $16 \mathrm{wk}$, the maximum failure loads in the HPC/PET group were about $502.3 \pm 20.0 \mathrm{~N}$ and $325.0 \pm 32.6 \mathrm{~N}$, respectively, which was significantly larger than that for PET group (4 wk: $182.7 \pm 14.5 \mathrm{~N}$ and $6 \mathrm{wk}: 142.0 \pm 5.3 \mathrm{~N}$, $p<0.01$ ) (Fig. 8A). However, the stiffness values of the limbs were not significantly different between PET grafts and HPC/PET grafts $(23.0 \pm 5.0 \mathrm{~N} / \mathrm{mm}$ vs. $18.3 \pm 3.5 \mathrm{~N} / \mathrm{mm})$. Notably, at week 16 , the stiffness value of the limbs with HPC/PET grafts was significantly greater than that of the limbs with PET grafts $(46.7 \pm 7.8 \mathrm{~N} / \mathrm{mm}$ vs. $27.3 \pm 4.5 \mathrm{~N} / \mathrm{mm}, P=0.020)$ (Fig. 8B).

3.7 Histological analysis 
The histological analysis was conducted to detect the details of the interface between implant grafts and bone. As shown in Fig. 9, Masson staining analysis showed that at 4 wk after implantation, only a small amount of collagen fibers was found in the graft-bone interface of PET grafts (Fig. 9A), whereas more abundant collagen fibers existed in the HPC/PET grafts (Fig. 9B). Most importantly, $16 \mathrm{wk}$ after implantation, the amount of collagen fibers increased in the PET grafts, but no new bone formation was observed (Fig. 9C). However, protruding new bone tissue emerged from the host bone to the graft in the HPC/PET grafts (Fig. 9D). These results suggest that HPC coated on the surface of PET could effectively induce the growth of new bone tissues in vivo.
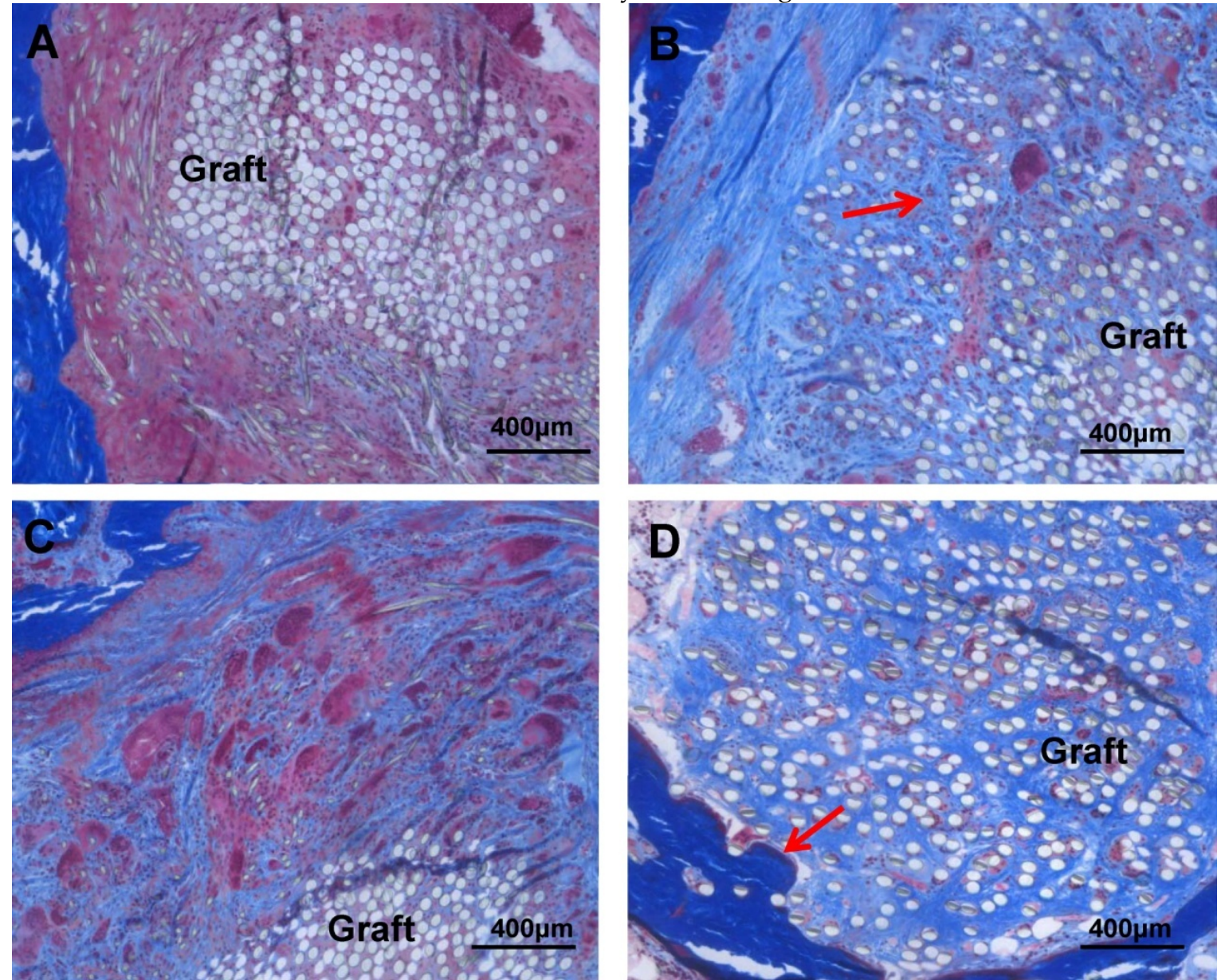

Fig. 9. Masson staining showing histological characteristics of graft-bone interface of the graft-bone interface of PET grafts and HPC/PET grafts 4 (A and B) and 16 (C and D) wk after surgery. Red arrow shows the collagen tissue in and around the graft (B), and the new bone (D). Graft, PET artificial ligament. Bar $=400 \mu \mathrm{m}$.

\subsection{Expression of BMP-7, COL3, OCN, RUNX2, TGF- $\beta 1$ and VEGF mRNA}

Table 2 show the mRNA expression results for genes related to the bone to tendon healing by RT-PCR analysis. As shown in Table 2, after $4 \mathrm{wk}$, it was observed that genes including BMP-7 and TGF- $\beta 1$ had significantly higher expression in the HPC/PET group compared with PET grafts. However, both COL3 and VEGF mRNA expression was significantly decreased. Furthermore, the mRNA levels of BMP-7 and OCN decreased with increasing time, while the mRNA expression levels of OCN and RUNX2 were not significantly different between the two groups.

At week 16, there was no significant difference in the mRNA expression levels of BMP-7, TGF- $\beta 1$, and VEGF between the two groups. Furthermore, with increasing time, the mRNA levels of BMP-7 and TGF- $\beta 1$ decreased but those of COL3, OCN, RUNX2 and VEGF increased (Table 2). Notably, COL3, OCN and RUNX2 mRNA expression were significantly higher in the HPC/PET group in the PET group. 
Table 2 mRNA levels for graft "ligamentization" related proteins in the PET grafts and HPC/PET grafts at 4 and 16 wk after surgery

\begin{tabular}{ccccccc}
\cline { 3 - 7 } & & \multicolumn{2}{c}{$4 \mathrm{wk}$} & \multicolumn{3}{c}{$16 \mathrm{wk}$} \\
\cline { 2 - 7 } PET & HPC/PET & P value & PET & HPC/PET & P value \\
\hline BMP-7 & $0.150 \pm 0.015$ & $0.527 \pm 0.074$ & 0.001 & $0.193 \pm 0.065$ & $1.283 \pm 0.055$ & 0.141 \\
TGF- $\beta 1$ & $0.024 \pm 0.003$ & $0.042 \pm 0.004$ & 0.004 & $0.030 \pm 0.013$ & $1.035 \pm 0.012$ & 0.691 \\
COL3 & $0.012 \pm 0.005$ & $0.024 \pm 0.004$ & 0.041 & $0.019 \pm 0.004$ & $1.036 \pm 0.007$ & 0.023 \\
VEGF & $0.003 \pm 0.001$ & $0.005 \pm 0.002$ & 0.048 & $0.004 \pm 0.001$ & $1.006 \pm 0.001$ & 0.132 \\
OCN & $0.493 \pm 0.130$ & $0.610 \pm 0.090$ & 0.271 & $0.493 \pm 0.131$ & $1.763 \pm 0.144$ & 0.032 \\
RUNX2 & $0.030 \pm 0.006$ & $0.041 \pm 0.009$ & 0.136 & $0.033 \pm 0.008$ & $1.067 \pm 0.011$ & 0.012 \\
\hline
\end{tabular}

Abbreviations: HPC, hydroxypropylcellulose; BMP-7, bone morphogenetic protein-7; TGF- $\beta 1$, transforming growth factor- $\beta 1$; COL3, type III collagen; VEGF, vascular endothelial growth factor; OCN, osteocalcin.

\section{Discussion}

Previously, some investigators have shown that a hydrophilic coating, such as putative HLA-DR-associated protein-RGD motif or fibronectin, greatly favors cell adhesion ${ }^{24-25}$. We also observed with SEM, in the present study, that many BMSCs, fibroblasts and RSC-364 cells adhered to the HPC/PET sheets while few cells adhered to the PET sheets after $7 \mathrm{~d}$ in culture. Furthermore, we found that after $7 \mathrm{~d}$ in culture, the above three kinds of cells, compared with those on the PET sheets, extensively adhered onto the HPC/PET sheets and exhibited polygonal shapes with multiple cellular synaptic contacts. The expression levels of integrin- $\beta 1$ on the cells on the HPC/PET sheets were also higher than those on the PET sheets. These findings indicate that the cells had poor adhesion to the PET sheets, while the HPC coating helped improve the adhesion of cells on PET fibers. A previous study showed that rough surfaces with intermediate hydrophilicity were favorable for cell growth ${ }^{26}$, which was supported by our observation in the present study that cell proliferation levels of BMSCs, fibroblasts and RSC-364 cells were all higher on the HPC/PET sheets, compared with those of the cells on the PET sheets at various time points in culture. As previously reported, hydrophilic surfaces induce and enhance osteogenic differentiation of $\mathrm{BMSCs}^{27}$. In the present study, the levels of ALP and OPN in BMSCs on the HPC/PET sheets were significantly higher than those in cells on the PET sheets at various time points after culture. Furthermore, western blotting also showed that the expression levels of BMP-2 and BMP-7 in BMSCs on the HPC/PET sheets were significantly higher than those on the PET sheets after $7 \mathrm{~d}$ in culture. All these findings are likely due to the induction of osteogenic differentiation of BMSCs by an organic and hydrophilic coating HPC. Therefore, the characteristics of the graft's surface have a strong influence on the attachment and growth of BMSCs and fibroblasts on ligament fibers and osteogenic differentiation of BMSCs after implantation.

Recently, large animal models of ACL reconstruction have been used to evaluate graft-bone healing. Previous studies have shown that hydrophilic biomaterials promote bone healing and osseointegration ${ }^{17},{ }^{28-32}$. Lang et al28 reported that, in a human bone defect model, the chemically modified moderately rough, hydrophilic (i.e., SLActive) implant surface performed better than moderately rough, hydrophobic (i.e., SLA) implant surface in the rate and degree of osseointegration during the early phase of healing (i.e., 2 and 4 wk). Our present study demonstrated that the HPC coating significantly improved the biomechanical properties of the PET artificial ligament graft in an ACL reconstruction model in beagle dogs, suggesting that the hydrophilic HPC coating enhances the healing of the PET graft in the bone tunnel. Li et al 17 evaluated a layer-by-layer chitosan-hyaluronic acid (LBL) coating on a PET artificial ligament in a rabbit extra-articular tendon-bone healing model and observed that new bone formation at the graft-bone interface $8 \mathrm{wk}$ after implantation was more evident in the LBL group than in the PET group. In our study, more collagen fibers were found at week 4 in the HPC/PET grafts than in the PET grafts. Moreover, we observed new bone tissues protruding from the host bone to the graft at 
week 16 in the HPC/PET grafts, but not in the PET grafts. Again, these findings are likely explained by the fact that the HPC coating significantly improves the hydrophilicity of the PET surface.

Synovium may be beneficial to ligament healing and postoperative rehabilitation after ACL and PCL reconstruction ${ }^{33-34}$. Ikeda et al ${ }^{35}$ found that in a canine tendon explant culture model, interpositional synovial grafts potentially accelerated tendon healing when implanted at the repair site. Our present study also observed a thicker synovium covering on the reconstructed graft in the articular cavity at week 16 in the HPC/PET grafts, compared with that in the PET grafts. Yang et al ${ }^{36}$ reported that the COL1-based fibrous capsule enhanced integration of tissue-engineered cartilage with native articular cartilage. Duan et al37demonstrated that in a canine model, a composite including COL1, based on a biphasic scaffold in combination with BMSCs, exhibited a great potential to repair a large osteochondral defect. In the present study, we observed that COL1 expression in the articular cavity was significantly higher in the HPC/PET grafts than that in PET grafts after implantation.

Previous studies have shown that several proteins and cell factors surrounding the graft, such as BMP-7, OCN, RUNX2 and TGF- $\beta 1$, favor osteogenesis. ${ }^{38-41}$ BMP-7 is an osteo-inductive morphogen that is known to be involved in embryonic skeletogenesis as well as in bone repair. Den Boer et al ${ }^{39}$ reported that in an experimental study, callus diameter, callus volume, and bone mineral content at the fracture site were significantly increased in BMP-7 groups, compared with the no-injection control group after surgery, suggesting that BMP-7 is able to accelerate physiologic fracture healing. Furthermore, $\mathrm{OCN}$, a bone-specific extracellular matrix protein, is a marker of mature osteoblasts. Verbicaro et al ${ }^{40}$ demonstrated that in rat and rabbit animal models, OCN immunoexpression was more evident during repair of critical-sized bone defects which were treated with subcutaneous adipose tissue. In addition, Wojtowicz et al ${ }^{41}$ reported that RUNX2 over-expression in bone marrow stromal cells accelerated bone formation in critical-sized femoral defects, and Tanaka et al ${ }^{42}$ reported that rhTGF- $\beta 1$ induced intramembranous ossification in neonatal rat parietal bone. Our present study further demonstrated that the mRNA levels of BMP-7 and TGF- $\beta 1$ at week 4 and of OCN and RUNX2 at week 16 were significantly upregulated in the HPC/PET grafts, compared with those in the PET grafts.

An increasing number of studies have shown that COL3 and VEGF enhance vascular proliferation and collagen remodeling ${ }^{43-44}$. COL3 plays a key role in tissue repair owning to its temporospatial expression during the healing. Miedel et al ${ }^{43}$ found that COL3 regulated osteoblast differentiation, the quality of cutaneous repair and trabecular bone. VEGF is a polypeptide that stimulates new blood vessel formation. Yoshikawa et al44 found that VEGF participates in the graft remodeling after ACL reconstruction especially in the early stage. Our present study also demonstrated that the mRNA levels of COL3 and VEGF in the early stage after implantation were significantly higher in the HPC/PET grafts than in the PET grafts. Moreover, COL3 mRNA expression at week 16 was also significantly higher in the HPC/PET grafts than in the PET grafts. These findings suggest that the enhanced hydrophilicity of the HPC/PET graft facilitates the "ligamentization" process.

\section{Conclusions}

A novel HPC-coated PET artificial ligament was successfully designed, prepared, and evaluated in vitro and in vivo. HPC coating significantly improved the biocompatibility of the PET artificial ligament and promoted graft-to-bone healing in ACL reconstruction. Our product offers a potential solution for overcoming the poor ligamentization of current PET artificial ligaments after implantation.

\section{Acknowledgments}

We are grateful to Professor Wei Shen, who works at College of Textiles, Donghua University, Shanghai, People's Republic of China, for offering the PET artificial ligament for beagle dogs ACL reconstruction. This work was supported by National Key R\&D Program of China (2016YFC1100300, 2017YFC0840100 and 2017YFC0840106), National Natural Science Foundation of China (NO. 81572108, NO. 81601879 and 
81611130221), Youth Elite Training Action Plan of Tongji University (NO. 2016KJ059), Medical and health science and technology project of Zhejiang Province (2015117887), the Key Clinical Medicine Center of Shanghai (2017ZZ01006), Sanming Project of Medicine in Shenzhen (SZSM201612078), Shanghai Rising-Star Project (18QB1400500), CAS Key Laboratory of Nano-Bio Interface(No:17NBI01) and the Introduction Project of Clinical Medicine Expert Team for Suzhou (SZYJTD201714). The authors thank Medjaden Bioscience Limited for assisting in preparation of this manuscript. Jun Chen and Nguyen T. K. Thanh thank the Royal Society for the funding. NTK Thanh thanks EPSRC and AOARD for funding.

\section{Conflicts of Interest}

The authors declare no conflict of interest.

\section{Author Contributions}

J. Yang, Y. Dong and J. Wang conceived and designed the experiments; J. Yang, J. Jiang, Y. Zhu, P. Zhang, T. Chen, W. Zhou and P. Wu performed the experiments; J. Yang, J. Jiang, Y. Zhu, and N. Tran analyzed the data; J. Chen and S. Chen contributed reagents/materials/analysis tools; J. Chen, S. Chen and N. T. K. Thanh wrote the paper.

\section{Reference:}

1. Spindler, K. P.; Wright, R. W., Anterior Cruciate Ligament Tear. N. Engl. J. Med. 2008, 359 (20), 2135-2142.

2. Frobell, R. B.; Roos, E. M.; Roos, H. P.; Ranstam, J.; Lohmander, L. S., A Randomized Trial of Treatment for Acute Anterior Cruciate Ligament Tears. N. Engl. J. Med. 2010, 363 (4), 331-342.

3. Prodromos, C. C.; Han, Y.; Rogowski, J.; Joyce, B.; Shi, K., A meta-analysis of the incidence of anterior cruciate ligament tears as a function of gender, sport, and a knee injury-reduction regimen. Arthroscopy-the Journal of Arthroscopic and Related Surgery 2007, 23 (12), 1320-1325.

4. Yilgor, C.; Huri, P. Y.; Huri, G., Tissue Engineering Strategies in Ligament Regeneration. Stem Cells Int 2012.

5. Legnani, C.; Ventura, A.; Terzaghi, C.; Borgo, E.; Albisetti, W., Anterior cruciate ligament reconstruction with synthetic grafts. A review of literature. Int. Orthop. 2010, 34 (4), 465-471.

6. Jia, Z.; Xue, C.; Wei, W.; Liu, T.; Xuan, H.; Xu, W., Clinical outcomes of anterior cruciate ligament reconstruction using LARS artificial graft with an at least 7-year follow-up. Medicine 2017, 96 (14), e6568.

7. Chen, J.; Gu, A.; Jiang, H.; Zhang, W.; Yu, X., A comparison of acute and chronic anterior cruciate ligament reconstruction using LARS artificial ligaments: a randomized prospective study with a 5-year follow-up. Arch. Orthop. Trauma Surg. 2015, 135 (1), 95-102.

8. Barbier, O.; Guérard, S.; Boisrenoult, P.; Thoreux, P., Biomechanical evaluation of four femoral fixation configurations in a simulated anterior cruciate ligament replacement using a new generation of Ligament Advanced Reinforcement System (LARSTM AC). Eur. J. Orthop. Surg. Traumatol. 2015, 25 (5), 905-911.

9. Parchi, P. D.; Gianluca, C.; Dolfi, L.; Baluganti, A.; Nicola, P.; Chiellini, F.; Lisanti, M., Anterior cruciate ligament reconstruction with LARS ${ }^{\mathrm{TM}}$ artificial ligament results at a mean follow-up of eight years. Int. Orthop. 2013, 37 (8), 1567-1574.

10. Li, H.; Yao, Z.; Jiang, J.; Hua, Y.; Chen, J.; Li, Y.; Gao, K.; Chen, S., Biologic Failure of a Ligament Advanced Reinforcement System Artificial Ligament in Anterior Cruciate Ligament Reconstruction: A Report of Serious Knee Synovitis. Arthroscopy 2012, 28 (4), 583-586.

11. Glezos, C. M.; Waller, A.; Bourke, H. E.; Salmon, L. J.; Pinczewski, L. A., Disabling synovitis associated with LARS artificial ligament use in anterior cruciate ligament reconstruction: a case report. Am. J. Sports Med. 2012, 40 (5), 1167. 
12. Gao, K.; Chen, S.; Wang, L.; Zhang, W.; Kang, Y.; Dong, Q.; Zhou, H.; Li, L., Anterior Cruciate Ligament Reconstruction With LARS Artificial Ligament: A Multicenter Study With 3- to 5-Year Follow-up. Arthroscopy the Journal of Arthroscopic \& Related Surgery 2010, 26 (4), 515-523.

13. Tiefenboeck, T. M.; Thurmaier, E.; Tiefenboeck, M. M.; Ostermann, R. C.; Joestl, J.; Winnisch, M.; Schurz, M.; Hajdu, S.; Hofbauer, M., Clinical and functional outcome after anterior cruciate ligament reconstruction using the LARS ${ }^{\mathrm{TM}}$ system at a minimum follow-up of 10years. Knee 2015, 22 (6), 565-568.

14. Gulotta, L. V.; Kovacevic, D.; Ying, L.; Ehteshami, J. R.; Montgomery, S.; Rodeo, S. A., Augmentation of tendon-to-bone healing with a magnesium-based bone adhesive. Am. J. Sports Med. 2008, 36 (7), 1290-1297.

15. Ma, Z.; Kotaki, M.; Yong, T.; He, W.; Ramakrishna, S., Surface engineering of electrospun polyethylene terephthalate (PET) nanofibers towards development of a new material for blood vessel engineering. Biomaterials 2005, 26 (15), 2527-2536.

16. Ai, C.; Cai, J.; Zhu, J.; Zhou, J.; Jiang, J.; Chen, S., Effect of PET graft coated with silk fibroin via EDC/NHS crosslink on graft-bone healing in ACL reconstruction. Rsc Advances 2017, 7 (81), 51303-51312.

17. Hong, L.; Chen, C.; Zhang, S.; Jia, J.; Tao, H.; Xu, J.; Sun, J.; Wei, Z.; Chen, S., The use of layer-by-layer self assembled coatings of hyaluronic acid and cationized gelatin to improve the biocompatibility of poly (ethylene terephthalate) artificial ligaments for the reconstruction of anterior cruciate ligament. Acta Biomater. 2012, 8 (11), 4007-4019.

18. Zhang, P.; Han, F.; Li, Y.; Chen, J.; Chen, T.; Zhi, Y.; Jiang, J.; Lin, C.; Chen, S.; Zhao, P., Local delivery of controlled-release simvastatin to improve the biocompatibility of polyethylene terephthalate artificial ligaments for reconstruction of the anterior cruciate ligament. Int. J. Nanomed. 2016, 11 (C), 465.

19. Yang, J.; Jiang, J.; Li, Y.; Li, H.; Jing, Y.; Wu, P.; Yu, D.; Chen, S., A new strategy to enhance artificial ligament graft osseointegration in the bone tunnel using hydroxypropylcellulose. Int. Orthop. 2013, 37 (3), 515-521.

20. Li, H.; Ge, Y.; Wu, Y.; Jiang, J.; Gao, K.; Zhang, P.; Wu, L.; Chen, S., Hydroxyapatite coating enhances polyethylene terephthalate artificial ligament graft osseointegration in the bone tunnel. Int. Orthop. 2011, 35 (10), 1561-1567.

21. Mezdour, S.; Lepine, A.; Erazo-Majewicz, P.; Ducept, F.; Michon, C., Oil/water surface rheological properties of hydroxypropyl cellulose (HPC) alone and mixed with lecithin: Contribution to emulsion stability. Colloids Surf., A 2017, 331 (1), 76-83.

22. Guan, Y.; Zhang, Y.; Zhou, T.; Zhou, S., Stability of hydrogen-bonded hydroxypropylcellulose/poly(acrylic acid) microcapsules in aqueous solutions. Soft Matter 2009, 5 (4), 842-849.

23. Huangfu, X.; Zhao, J., Tendon-bone healing enhancement using injectable tricalcium phosphate in a dog anterior cruciate ligament reconstruction model. Arthroscopy 2007, 23 (5), 455-462.

24. Lu, H. H.; Jr, C. J.; Manuel, S.; Freeman, J. W.; Attawia, M. A.; Ko, F. K.; Laurencin, C. T., Anterior cruciate ligament regeneration using braided biodegradable scaffolds: in vitro optimization studies. Biomaterials 2005, 26 (23), 4805-4816.

25. Yu, D.; Zhang, Q.; Li, Y.; Jia, J.; Chen, S., Enhancement of Tendon-Bone Healing for Anterior Cruciate Ligament (ACL) Reconstruction Using Bone Marrow-Derived Mesenchymal Stem Cells Infected with BMP-2. Int. J. Mol. Sci. 2012, 13 (10), 13605-13620.

26. Shen, H.; Hu, X.; Yang, F.; Bei, J.; Wang, S., Combining oxygen plasma treatment with anchorage of cationized gelatin for enhancing cell affinity of poly(lactide-co-glycolide). Biomaterials 2007, 28 (29), 4219-4230. 
27. Park, H.; Choi, B.; Nguyen, J.; Fan, J.; Shafi, S.; Klokkevold, P.; Min, L., Anionic Carbohydrate-containing Chitosan Scaffolds for Bone Regeneration. Carbohydr. Polym. 2013, 97 (2), 587-596. 28. Lang, N. P.; Salvi, G. E.; Huynh-Ba, G.; Ivanovski, S.; Donos, N.; Bosshardt, D. D., Early osseointegration to hydrophilic and hydrophobic implant surfaces in humans. Clin. Oral Implants Res. 2015, 22 (4), 349-356.

29. Chou, Y. C.; Yeh, W. L.; Chao, C. L.; Hsu, Y. H.; Yu, Y. H.; Chen, J. K.; Liu, S. J., Enhancement of tendon-bone healing via the combination of biodegradable collagen-loaded nanofibrous membranes and a three-dimensional printed bone-anchoring bolt. Int. J. Nanomed. 2016, 11, 4173-4186.

30. Petzold, C.; Monjo, M.; Rubert, M.; Reinholt, F. P.; Gomezflorit, M.; Ramis, J. M.; Ellingsen, J. E.; Lyngstadaas, S. P., Effect of proline-rich synthetic peptide-coated titanium implants on bone healing in a rabbit model. Int. J. Oral Maxillofac. Implants 2013, 28 (6), e547.

31. Tu, Y.; Chen, C.; Li, Y.; Hou, Y.; Huang, M.; Zhang, L., Fabrication of nano-hydroxyapatite/chitosan membrane with asymmetric structure and its applications in guided bone regeneration. Biomed. Mater. Eng. 2017, 28 (3), 223-233.

32. Cho, Y.; Hong, J.; Ryoo, H.; Kim, D.; Park, J.; Han, J., Osteogenic Responses to Zirconia with Hydroxyapatite Coating by Aerosol Deposition. J. Dent. Res. 2015, 94 (3), 491-9.

33. Wang, C. J.; Chan, Y. S.; Weng, L. H., Posterior cruciate ligament reconstruction using hamstring tendon graft with remnant augmentation. Arthroscopy 2005, 21 (11), 1401.e1-1401.e3.

34. Zhang, Y.; Huang, W.; Jiang, J.; Xie, J.; Xu, C.; Wang, C.; Yin, L.; Yang, L.; Zhou, K.; Chen, P., Influence of TNF- $\alpha$ and biomechanical stress on matrix metalloproteinases and lysyl oxidases expressions in human knee synovial fibroblasts. Knee Surg. Sports Traumatol. Arthrosc. 2014, 22 (9), 1997-2006.

35. Ikeda, J.; Zhao, C.; Moran, S. L.; An, K. N.; Amadio, P. C., The Effects of Synovial Interposition on Healing In a Canine Tendon Explant Culture Model. J. Hand Surg. Am. 2010, 35 (7), 1153-1159.

36. Yang, Y. H.; Ard, M. B.; Halper, J. T.; Barabino, G. A., Type I Collagen-Based Fibrous Capsule Enhances Integration of Tissue-Engineered Cartilage with Native Articular Cartilage. Ann. Biomed. Eng. 2014, 42 (4), 716-726.

37. Duan, X.; Zhu, X.; Dong, X.; Yang, J.; Huang, F.; Cen, S.; Leung, F.; Fan, H.; Xiang, Z., Repair of large osteochondral defects in a beagle model with a novel type I collagen/glycosaminoglycan-porous titanium biphasic scaffold. Mater. Sci. Eng. C 2013, 33 (7), 3951-3957.

38. Lesaichot, V.; Leperlier, D.; Viateau, V.; Richarme, D.; Petite, H.; Sailhan, F., The influence of Bone Morphogenic Protein-2 on the consolidation phase in a distraction osteogenesis model. Injury 2011, 42 (12), 1460-1466.

39. den Boer, F. C.; Bramer, J. A.; Blokhuis, T. J.; Van Soest, E. J.; Jenner, J. M.; Patka, P.; Bakker, F. C.; Burger, E. H.; Haarman, H. J., Effect of recombinant human osteogenic protein-1 on the healing of a freshly closed diaphyseal fracture. Bone 2002, 31 (1), 158-164.

40. Verbicaro, T.; Giovanini, A. F.; Zielak, J. C.; Baratto, F. F.; de Araujo, M. R.; Deliberador, T. M., Osteocalcin immunohistochemical expression during repair of critical-sized bone defects treated with subcutaneous adipose tissue in rat and rabbit animal model. Braz. Dent. J. 2013, 24 (6), 559.

41. Wojtowicz, A. M.; Templeman, K. L.; Hutmacher, D. W.; Guldberg, R. E.; García, A. J., Runx2 Overexpression in Bone Marrow Stromal Cells Accelerates Bone Formation in Critical-Sized Femoral Defects. Tissue Eng., Part A 2010, 16 (9), 2795.

42. Tanaka, T.; Taniguchi, Y.; Gotoh, K.; Satoh, R.; Inazu, M.; Ozawa, H., Morphological study of recombinant human transforming growth factor beta 1-induced intramembranous ossification in neonatal rat parietal bone. Bone 1993, 14 (2), 117-123. 
43. Miedel, E. L.; Brisson, B. K.; Hamilton, T.; Gleason, H.; Swain, G. P.; Lopas, L.; Dopkin, D.; Perosky, J. E.; Kozloff, K. M.; Hankenson, K. D., Type III collagen modulates fracture callus bone formation and early remodeling. J. Orthop. Res. 2015, 33 (5), 675-684.

44. Yoshikawa, T.; Tohyama, H., H; Matsumoto, H.; Toyama, Y.; Yasuda, K., Expression of vascular endothelial growth factor and angiogenesis in patellar tendon grafts in the early phase after anterior cruciate ligament reconstruction. Knee Surg. Sports Tr. A. 2006, 14 (9), 804. 